\title{
Estimulação Hormonal, Punção Folicular Transvaginal e Avaliação Ovocitária em Bezerras Pré-Púberes da Raça Nelore (Bos taurus indicus) ${ }^{1}$
}

\author{
Marilia Viviane Snel-Oliveira ${ }^{2}$, Daniela Costa Pereira ${ }^{3}$, Danilo Malagoli Junior ${ }^{4}$, Rodolfo Rumpf ${ }^{5}$
}

RESUMO - O uso de bezerras pré-púberes como doadoras de ovócitos oferece um considerável potencial para acelerar o ganho genético através da diminuição do intervalo de gerações, além de incrementar o aproveitamento de fêmeas bovinas de alto valor genético. Os objetivos deste estudo foram analisar o efeito da idade e do tratamento estimulatório com gonadotrofinas sobre a resposta folicular, a taxa de recuperação, a produção e a avaliação morfológica de ovócitos colhidos em bezerras da raça nelore (B. t. indicus) a partir de 10 meses de idade. As bezerras foram distribuídas aleatoriamente em três grupos $(n=4)$ e receberam tratamentos hormonais distintos, repetidos nos mesmos animais nos três períodos, aos 10, 11 e 12 meses de idade. No dia zero (D0), todas as bezerras receberam um implante intravaginal de progesterona e, em D1, $2 \mathrm{mg}$ de benzoato de estradiol intramuscular (im). Em D6, deu-se início à diferenciação dos tratamentos (T): T1 - 120 UI de FSH, divididos em quatro aplicações im a cada 12 horas; T2 - 120 UI de FSH, em uma única aplicação subcutânea (sc), mais 300 UI de eCG sc, T3 - sem tratamento estimulatório. No D8, o implante de progesterona foi retirado e a punção folicular transvaginal, guiada por ultra-som (PFTV), foi realizada no D9. Dos 402 ovócitos coletados, 53 foram desnudados e medidos, incluindo a zona pelúcida, encontrando-se um diâmetro médio de 153,38 $(8,06) \mu \mathrm{m}$. Não houve diferença significativa entre os tratamentos e entre as idades para quantidade de folículos $\geq 3 \mathrm{~mm}$, de ovócitos recuperados e de ovócitos viáveis. A média de ovócitos recuperados e ovócitos viáveis para cada tratamento foi: T1- $11,08(6,20)$ e 59,58\% (25,80), T2- $12,75(8,04)$ e $66,02 \%(36,71)$, T3 - 9,66 (8,15) e 51,51\% (30,69), respectivamente. O valor médio da taxa de recuperação foi de 78,70\%. O uso de FSH e FSH + eCG não aumentou significantemente a quantidade de folículos $\geq 3 \mathrm{~mm}$ no momento da PFTV, assim como dos ovócitos recuperados e viáveis aos 10,11 e 12 meses de idade.

Palavras chave: bezerras pré-púberes, estimulação hormonal, ovócitos, punção folicular zebuíno

\section{Hormonal Stimulation and Ultrasound Guided Transvaginal Follicle Aspiration and Oocyte Recovery in Nelore (Bos taurus indicus) Prepubertal Calves}

\begin{abstract}
The use of pre-pubertal calves as oocyte donors offers a considerable potential to increase the genetic gain lowering the generations interval, as well as increase the usage of bovine females with high genetic value. The objectives of this study were to analyze the effect of age and of the stimulatory treatment with gonadotropins in the follicular response, the recovery rate, quantity and quality of oocytes of the prepubertal Nelore (B. $t$. indicus) calves. Twelve Nelore prepubertal females were stimulated with gonadotrophin hormone and were submitted to ultrasound guided transvaginal oocyte recovery (OPU) at 10, 11 and 12 months of age. Before OPU the females were randomly distributed into three treatment groups $(n=4)$, which consist of different protocols of ovarian super stimulation. For all treatments the animals on day zero (D0) received one intravaginal progesterone implant (CIDR-G®) and on

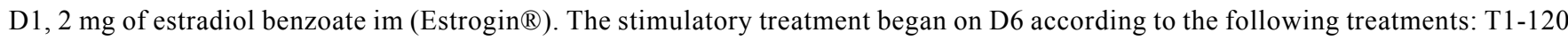
IU of FSH (Foltropin ${ }^{\circledR}$ ), divided in 4 im injections with 12 hours interval; T2-120 IU of FSH in one sc application plus $300 \mathrm{IU}$ of eCG (Folligon ${ }^{\circledR}$ ) on D6; T3 - without stimulatory treatment (control). At D8 the CIDR-G ${ }^{\circledR}$ was removed and OPU was performed on D9. Among the 402 oocytes retrieved, 53 were denuded and measured (including the pellucid zone) where a mean diameter of $153.38 \pm 8.06 \mu \mathrm{m}$ was obtained. No significant difference was detected among treatments and among ages for the quantity of follicles $\geq 3 \mathrm{~mm}$, quantity of oocytes recovery and quantity of oocytes morphologically viable. The mean of recovery oocytes and viable oocytes for each treatment was T1- 11.08 (6.20) and 59.58\% (25.80), T2 - $12.75(8.04)$ and 66.02\% (36.71), T3 - $9.66(8.15)$ and 51.51\% (30.69), respectively. The mean value of recovery rate was $78.70 \%$. The use of FSH or FSH + eCG does not increase significantly the amount of follicles $\geq 3 \mathrm{~mm}$ at the OPU moment, as well as the total and viable oocytes by 10,11 and 12 months of age.
\end{abstract}

Key Words: hormonal stimulation, pre-pubertal calf, oocyte, OPU, Zebu

\footnotetext{
1 Parte da Dissertação de Mestrado apresentada à Universidade de Brasília pelo primeiro autor.

2 Médica Veterinária, MSc. Professora dos Departamentos de Medicina Veterinária e Zootecnia da UPIS - Faculdades Integradas BR 020, Km 12, DF 335, Km 4,8. Brasília - DF. E.mail: marilia@upis.br

3 Médica Veterinária, autônoma.

${ }^{4}$ Médico Veterinário, Pie Medical - GE.

5 Médico Veterinário, Doutor, Pesquisador da EMBRAPA-CENARGEN. E.mail: rodolfo@cenargen.embrapa.br
} 


\section{Introdução}

O uso de doadoras pré-púberes em programas de transferência de embriões (TE) oferece um considerável potencial para acelerar o ganho genético em um rebanho de animais domésticos, através da diminuição do intervalo de gerações (IG) (Lohuis, 1995).

Os folículos aparecem no ovário do feto bovino entre 220 e 240 dias de gestação. Ao nascer, a bezerra possui de 75.000 a 300.000 ovócitos; apesar de nenhum folículo ser visível macroscopicamente ao nascimento, aos 5 dias de vida já há folículos de $5 \mathrm{~mm}$. Devido à senescência no decorrer da vida dos animais, os ovários de bezerras contêm muito mais folículos visíveis na superfície do que os de vacas (Erickson $1966 \mathrm{ab).} \mathrm{Estudando} \mathrm{bezerras} \mathrm{holandesas,}$ Erickson (1966b) e Desjardins \& Hafs (1969) observaram que os folículos atingem uma quantidade máxima aos 4 a 6 meses de idade, decrescem até os 7 a 8 meses (puberdade) e permanecem constante desta idade até 10 a 14 anos. Os ovócitos podem ser considerados como as células mais raras existentes no organismo, pois além de seu estoque não ser renovável, em condições naturais, menos de $0,1 \%$ dos folículos chegam a ovular (Gosden, 1998).

A produção de embriões de bezerras pré-púberes através de múltiplas ovulações e transferência de embriões (MOTE) não tem alcançado bons resultados, defrontando-se com diversas limitações. Em vista disso, as pesquisas têm-se voltado para aplicação da técnica de colheita de ovócitos de animais vivos, punção folicular via transvaginal, guiada por ultra-som (PFTV), associada às técnicas de produção de embriões in vitro. Desse modo, as bezerras pré-púberes podem ser utilizadas em programas de melhoramento animal, contribuindo para aumentar o ganho genético por diminuir o IG e por proporcionar um maior aproveitamento dos inúmeros ovócitos disponíveis, produzindo-se mais filhos de um animal de provável alto valor genético.

Têm-se obtido embriões viáveis, gestações e bezerros nascidos, provenientes de ovócitos de bezerras pré-púberes em diferentes idades (Kajihara et al., 1991; Armstrong et al., 1992; Revel et al., 1995; Fry et al., 1998; Bols et al., 1999; Brogliatti et al., 1999; Malard, 2000; Taneja et al., 2000). Entretanto, os ovócitos coletados de bezerras são menos competentes para se desenvolver em embriões que os de vacas (Seidel et al., 1971; Palma et al., 1993; Lévesque \& Sirard, 1994; Revel et al., 1995; Damiani et al., 1995,
1996; Khatir et al., 1996; Kuwer et al., 1999); esta competência parece aumentar com a idade da bezerra (Presicce et al., 1997), embora Armstrong et al. (1992), Irvine et al. (1993) e Earl et al. (1998) tenham obtido resultados semelhantes para animais prépúberes e adultos.

Para aumentar o número de ovócitos viáveis por coleta, o desenvolvimento folicular tem sido estimulado usando-se hormônios gonadotróficos, aplicados em diferentes esquemas, dosagens e produtos, com resultados variáveis e pouco conclusivos (Kajihara et al., 1991; Stubbings et al., 1992, 1993; Armstrong et al., 1992, 1994; Revel et al., 1995; Looney et al., 1995; Damiani et al., 1996; Tervit et al., 1997; Presicce et al., 1997; Fry et al., 1998; Malard, 2000; Taneja et al., 2000). Já outros estudos têm optado por coletas mais freqüentes sem a estimulação hormonal (Rick et al., 1996; Presicce et al., 1997; Brogliatti et al., 1999; Majerus et al., 1999).

Apesar de a subespécie Bos taurus indicus (B. Indicus) atingir a puberdade somente aos 19,9 a 28,7 meses de idade (Silva \& Romano, 1991; Restle et al., 1999; Rodrigues et al., 1999) e de sua grande importância econômica e social, especialmente nos trópicos, esses somente foram estudados por Maclellan et al. (1998ab), Malard (2000) e Brogliatti et al. (1999). Os demais trabalhos utilizam Bos taurus taurus (B. Taurus), especialmente as raças Holandesa e Simental. Como há nos animais adultos dessas duas subespécies diferenças na dinâmica folicular, estro e ovulação (Barros et al., 1995), é necessário considerar estas diferenças ao comparar os resultados deste com os de outros trabalhos.

Com o objetivo de avaliar o uso de doadoras prépúberes da raça Nelore (B. indicus), verificaram-se o efeito da idade e do tratamento estimulatório com gonadotrofinas sobre o número de folículos, a taxa de recuperação, a produção e a qualidade morfológica de ovócitos colhidos pela punção folicular por via transvaginal, orientada pela ultra-sonografia.

\section{Material e Métodos}

Este experimento foi desenvolvido no Campo Experimental Sucupira da Embrapa-Cenargen, em Brasília - DF, Brasil. Foram utilizadas 12 bezerras zebuínas (B. indicus) da raça Nelore, com peso médio de $167,5 \mathrm{~kg}$ (142 a $205 \mathrm{~kg}$ ) e 10 meses de idade, oriundas de um rebanho da região. Estes animais foram desmamados em fevereiro de 2000, com 6 
meses de idade, ocasião em que foram transportados para o local do experimento.

O estudo iniciou-se no mês de junho, quando as bezerras recebiam um suplemento concentrado à base de milho, além de pasto. Após o término do primeiro, de três períodos do experimento, os animais passaram a receber também silagem de milho, pois já não havia mais pastagem disponível suficiente devido à estiagem. Esta alimentação foi mantida até o final do experimento. As bezerras foram distribuídas aleatoriamente em 3 grupos $(n=4)$ e receberam tratamentos hormonais distintos, repetidos nos mesmos animais nos 3 períodos, aos 10, 11 e 12 meses de idade.

As PFTV foram realizadas em $3\left(1^{\circ}\right.$ período) ou 2 dias $\left(2^{\circ}\right.$ e $3^{\circ}$ períodos $)$ consecutivos de maneira que todos os tratamentos estivessem representados em cada dia de colheita, sendo respeitados os intervalos da estimulação hormonal às punções. Todos os animais foram submetidos às mesmas condições de manejo.

Tratamento hormonal: no dia zero (D0), foram realizados exames ovarianos com o uso de um aparelho de ultra-som (Aloka SSD-500, Overseas Monitor Corporation, Richmond, B.C.), equipado com um transdutor transretal de $5,5 \mathrm{mHZ}$, e os folículos $\geq 3$ $\mathrm{mm}$ foram contados por análise de imagem em tempo real. As bezerras receberam um implante intravaginal de progesterona para cabras (CIDR-G $\left.{ }^{\circledR}\right)$ e, em D1, foram aplicados, via intramuscular (im), $2 \mathrm{mg}$ de benzoato de estradiol (BE) (Estrogin $\AA$ ), com o intuito de sincronizar o início de uma nova onda folicular. Em D6, os ovários foram novamente examinados e os folículos $\geq 3 \mathrm{~mm}$ foram contados, utilizando-se o mesmo equipamento de ultra-som. Neste momento, deu-se início à diferenciação dos tratamentos: tratamento 1 - 120 UI de hormônio folículo estimulante (FSH) (Folltropin $\left.{ }^{\circledR}\right)$, dividido em quatro aplicações im a cada 12 horas; tratamento 2 - 120 UI de FSH (Folltropin $\left.{ }^{\circledR}\right)$, em uma única aplicação subcutânea (sc), mais 300 UI de gonadotrofina coriônica de égua (eCG) (Foligon $\left.{ }^{\circledR}\right)$ sc, tratamento 3 - controle - sem tratamento estimulatório. No D8, o CIDR-G® foi retirado e a PFTV foi realizada no D9.

Colheita dos ovócitos: a punção folicular foi realizada via transvaginal orientada por aparelho de ultra-som, conforme descrito por Pieterse et al. (1988), utilizando-se o equipamento General Eletric $\AA$, com transdutor microconvexo eletrônico, endocavitário, de $6,5 \mathrm{mHz}$, de banda larga de fre- qüência, com guia de biópsia, ligado à bomba de vácuo constante. $\mathrm{O}$ cabo do transdutor humano teve seu ângulo desfeito, aumentando-se, dessa forma, seu comprimento e viabilizando o seu uso nos animais jovens.

Os ovários foram localizados e fixados manualmente via transretal e os folículos $\geq 3 \mathrm{~mm}$ contados. A agulha (Frydman ${ }^{\circledR}$ ) de $17 \mathrm{G} \mathrm{com} 30 \mathrm{~cm}$ de comprimento foi previamente adaptada, retirando-se o conector de plástico para melhor conexão com mangueira de silicone do sistema de vácuo e soldado a ela um indicador da posição do bisel. A aspiração foi realizada sob pressão contínua equivalente a um fluxo de 10 a $15 \mathrm{ml}$ de água/minuto.

O conteúdo folicular aspirado foi colhido em tubo cônico, contendo solução salina, fosfatada e tamponada de Dulbecco (D'PBS), com 10\% de soro fetal bovino (SFB) (Gibco), $500 \mu 1$ de gentamicina (Sigma) e $32 \mathrm{mg}$ de heparina (Sigma) em $50 \mathrm{~mL}$ de D'PBS e filtrado em uma membrana de $75 \mu \mathrm{m}$ (Filtro Em-Con). O filtro com os ovócitos retidos foi lavado com D'PBS sem heparina e os ovócitos foram selecionados em um estereomicroscópio com aumento de até 120 vezes.

Classificação dos complexos cumulus ovócito (COC): a classificação dos COC seguiu os mesmos parâmetros utilizados por Malard (2000), ou seja: QI - ovócitos com citoplasma homogêneo e 4 ou mais camadas de células do cumulus; QII - ovócitos com citoplasma apresentando alguma granulação e/ou menos de 4 camadas de células de cumulus; incluindo os parcialmente desnudos; QIII - ovócitos com citoplasma homogêneo ou apresentando alguma granulação, mas com as células do cumulus expandidas; QIV - ovócitos com citoplasma homogêneo ou apresentando alguma granulação, mas desnudos; QV - ovócitos degenerados. Os ovócitos classificados como QI, QII e QIII foram considerados viáveis. O total de ovócitos colhidos QI, QII, QIII, QIV e QV será referido a seguir como ovócitos recuperados.

Tamanho dos ovócitos: cinqüenta e três ovócitos foram desnudados e tiveram seu diâmetro medido, incluindo a zona pelúcida, por meio de um microscópio de luz invertida, com uma ocular própria com escala previamente calibrada com um micrômetro.

Análises estatísticas: considerando que as mensurações nas mesmas unidades experimentais foram realizadas ao longo do tempo e que isto implica a violação do princípio de independência entre as observações, além de se querer discutir o efeito da 
variabilidade temporal do hormônio, utilizou-se a Análise de Variância com Medidas Repetidas no Tempo para a análise do número de folículos, do número de ovócitos recuperados, da porcentagem de ovócitos viáveis e da taxa de recuperação. Quando a hipótese de esfericidade dos componentes ortogonais foi aceita, foram utilizados os resultados do teste univariado; caso contrário, do teste multivariado (Crowder \& Hand, 1996; Girden, 1992). Foi realizada uma análise de correlação de Pearson para estabelecer se havia relação entre o número de folículos antes da primeira sincronização e o número de ovócitos colhidos nas três idades e se havia relação entre o número de folículos $\geq 3 \mathrm{~mm}$ a serem aspirados e o número de ovócitos recuperados e ovócitos viáveis colhidos nas três idades.

\section{Resultados e Discussão}

O escore corporal dos animais ao longo do experimento se manteve entre 2,5 e 3,0 em uma escala de 0 a 5 . O peso médio variou de $167,5 \mathrm{~kg}(142 \mathrm{a} 205 \mathrm{~kg})$ aos 10 meses e $203,16 \mathrm{~kg}(157 \mathrm{a} 245 \mathrm{~kg})$ aos 12 meses, apresentando um ganho médio diário de $0,424 \mathrm{~kg}$.

Resposta folicular: o número de folículos $\geq 3$ $\mathrm{mm}$, nos diferentes tratamentos e nos diferentes períodos, está apresentado na Tabela 1.

Observou-se um efeito de interação entre o tratamento e a idade no número de folículos $\geq 3 \mathrm{~mm}$ no momento da PFTV $(F=3,08 ; p<0,05)$. A contagem do número de folículos está sujeita a falhas, o que explicaria o fato de ter havido interação tratamento e tempo e não ter havido essa interação na quantidade de ovócitos recuperados e na quantidade de ovócitos viáveis. Dois fatores poderiam provocar essa falha na contagem. Primeiro, os folículos foram contados momentos antes e não durante a PFTV. Desse modo, pode-se ter deixado de contar folículos $\geq 3 \mathrm{~mm}$ que ficaram ocultos por folículos maiores. Segundo, na contagem dos folículos, foram computados somente os maiores ou iguais a $3 \mathrm{~mm}$, mas, como se trabalhou com bomba de vácuo de aspiração contínua, folículos menores foram, provavelmente, também puncionados.

Não houve diferenças significativas no número de folículos $\geq 3 \mathrm{~mm}$ entre os tratamentos e entre as idades, como demonstrado na tabela 1. Este resultado difere do encontrado por Earl et al. (1998) que, estudando bezerras holandesas de 4 a 12 semanas de idade, obtiveram um aumento progressivo no número de folículos $>3 \mathrm{~mm}$, conforme aumentava a idade das bezerras, havendo uma diferença significativa entre 4 a 6 semanas e 10 a 12 semanas de idade. Além disso, esses autores constataram que a estimulação com FSH aumentou 3 vezes o número de folículos visíveis (>3 mm), concluindo que a estimulação não só aumenta o tamanho dos folículos visíveis, como também estimula o recrutamento de folículos não visíveis antes da estimulação.

Adams et al. (1996) também obtiveram maior número de folículos em bezerras estimuladas às 14 semanas do que as 6 semanas. Essas observações coincidem com as de Desjardins \& Hafs (1969), que

Tabela 1 - Valores médios e desvios-padrão (DP) da quantidade de folículos $\geq 3 \mathrm{~mm}$ nas três idades (10, 11 e 12 meses) para cada tratamento (FSH; FSH + eCG e controle)

Table 1 - Means and standard deviation(SD) for the quantity of follicles $\geq 3 \mathrm{~mm}$ in the 3 ages $(10,11,12$ months) to each treatment (FSH; FSH + eCG and control)

\begin{tabular}{lccc}
\hline $\begin{array}{l}\text { Tratamento } \\
\text { Treatment }\end{array}$ & $\begin{array}{c}10 \text { meses média (DP) } \\
10 \text { months mean }(S D)\end{array}$ & $\begin{array}{c}11 \text { meses média (DP) } \\
11 \text { months mean }(S D)\end{array}$ & $\begin{array}{c}12 \text { meses média }(\mathrm{DP}) \\
12 \text { months mean }(S D)\end{array}$ \\
\hline $\mathrm{FSH}^{1}$ & $11,75(4,573)$ & $13,75(2,362)$ & $12,50(4,434)$ \\
FSH & $18,50(11,902)$ & $16,75(9,742)$ & $11,00(8,041)$ \\
$\mathrm{FSH}+\mathrm{eCG}^{2}$ & $10,00(1,633)$ & $13,25(7,455)$ & $8,00(4,690)$ \\
FSH $+e C G$ & Controle & & \\
Control & & & \\
\hline
\end{tabular}

Análise de variância com medidas repetidas no tempo (repeated measure analysis of variance), $\mathrm{p}>0,05$

1120 UI de FSH (Folltropin $\left.{ }^{\circledR}\right)$, divididos em quatro aplicações im a cada 12 horas, iniciado no D6 (120 IU of FSH (Folltropin $\AA$ ), divided into four im applications every 12 hours, starting on D6).

2120 UI de FSH (Folltropin $\left.{ }^{\circledR}\right)$, em uma única aplicação subcutânea (sc), mais 300 UI de eCG (Foligon $\left.{ }^{\circledR}\right)$ Sc, no D6 (120IU of FSH (Folltropin $\left.{ }^{\circledR}\right)$, subcutaneously (sc) in one single administration, plus 300 IU of sc eCG (Foligon $\left.{ }^{\circledR}\right)$, on D6).

3 Sem tratamento estimulatório (control - no stimulating treatment). 
estudaram bezerras holandesas do nascimento até 12 meses de idade. Eles verificaram que o número de folículos visíveis aumenta do nascimento até 4 meses, decresce de 4 a 8 meses, e permanece relativamente constante da puberdade (7-8 meses) em diante. A conhecida diferença de idade para atingir a puberdade entre um bovino Nelore e um bovino Holandês sugere haver grande diferença fisiológica entre eles aos $10 \mathrm{a}$ 12 meses e, dessa forma, estas comparações não podem ser feitas. Isto fica claro, quando se observa que Malard (2000), trabalhando com bezerras Nelore, não encontrou diferença significativa entre estimuladas e não estimuladas aos 2 e 4 meses de idade.

Número de ovócitos recuperados: neste experimento não se encontrou diferença significativa no número de ovócitos recuperados entre 10,11 ou 12 meses de idade, tanto nos tratamentos com estimulação hormonal (FSH e FSH + eCG) como no sem estimulação (controle) $(p>0,05)$. Este resultado foi encontrado tanto na análise univariada quanto na análise multivariada. Possivelmente, isto se deve ao fato de as bezerras Nelore serem mais tardias, entrando na puberdade entre 23,3 a 25,1 meses de idade (Silva \& Romano, 1991; Restle et al., 1999); deste modo, não há uma diferença fisiológica muito grande entre 10 e 12 meses de idade (Tabela 2). Por outro lado, é possível que os tratamentos de estimulação hormonal utilizados não tenham sido eficientes.

Entretanto, Rick et al. (1996), Presicce et al. (1997) e Majerus et al. (1999), trabalhando com bezerras Holandesas entre 6 e 12 meses de idade, verificaram que o número de ovócitos recuperados decresceu gradualmente enquanto aumentava a idade das bezerras não estimuladas e a puberdade era atingida.

Da mesma forma como o constatado por Irvine et al. (1993); Armstrong et al. (1994); Brogliatti \& Adams (1996), também não houve diferença entre estimular com múltiplas injeções de FSH ou com uma dose de FSH acompanhada de eCG.

Armstrong et al. (1997) obtiveram maior número de folículos retirando o dispositivo de progesterona 48 horas antes da PFTV do que quando foi retirado após a colheita. Como neste trabalho não houve diferença entre os animais estimulados com gonadotrofina e o controle, é possível que a retirada do CIDR-G 24 horas antes da PFTV tenha estimulado todos da mesma maneira.

Os resultados da bibliografia demonstram que a estimulação hormonal em bezerras pré-púberes é uma questão aberta e que muitos estudos deverão ser conduzidos parase chegar a um protocolo adequado (Armstrong et al, 1994; Brogliatti \& Adams, 1996; Armstrong et al, 1997; Presicce et al, 1997; Malard, 2000).

Taxa de recuperação: não houve diferença significativa na taxa de recuperação (TR) entre os tratamentos e entre as idades $(p>0,05)$, mas houve grande variação individual na taxa de recuperação. $\mathrm{O}$ valor médio da taxa de recuperação deste experimento foi de $78,70 \%$, um valor satisfatório quando se compara com trabalhos realizados em bezerras holandesas, a partir de 6 semanas até 15 meses de idade, nos quais as TR têm variado de 37 a 94,61\% (Presicce et al. 1995, 1997; Adams et al. 1996; Brogliatti \& Adams, 1996; Rick et al., 1996). Dessa forma, pode-se considerar eficiente o sistema utilizado.

Devido ao fato de a bomba de vácuo utilizada ter

Tabela 2 - Valores médios e desvios-padrão (DP) da quantidade de ovócitos recuperados nas três idades (10, 11 e 12 meses) para cada tratamento (FSH; FSH + eCG e controle)

Table 2 - Means and standard deviation (SD) of the quantity of oocyte recovery in the 3 ages (10, 11, 12 months) to each treatment (FSH; FSH + eCG and control)

\begin{tabular}{|c|c|c|c|c|}
\hline $\begin{array}{l}\text { Tratamento } \\
\text { Treatment }\end{array}$ & $\begin{array}{l}10 \text { meses média (DP) } \\
10 \text { months mean (SD) }\end{array}$ & $\begin{array}{l}11 \text { meses média (DP) } \\
11 \text { months mean (SD) }\end{array}$ & $\begin{array}{l}12 \text { meses média (DP) } \\
12 \text { months mean (SD) }\end{array}$ & $\begin{array}{l}\text { Média total (DP) } \\
\text { Total mean (SD) }\end{array}$ \\
\hline $\begin{array}{l}\mathrm{FSH}^{1} \\
\mathrm{FSH}\end{array}$ & $10,50(6,137)$ & $15,00 \quad(8,831)$ & $7,75 \quad(5,737)$ & $11,08(6,200)$ \\
\hline $\begin{array}{l}\mathrm{FSH}+\mathrm{eCG}^{2} \\
F S H+e C G\end{array}$ & $11,75(10,404)$ & $10,50(8,386)$ & $16,00(24,166)$ & $12,75(8,040)$ \\
\hline $\begin{array}{l}\text { Controle } \\
\text { Control }\end{array}$ & $12,25(8,655)$ & $9,25 \quad(10,688)$ & $7,50 \quad(5,802)$ & $9,66 \quad(8,151)$ \\
\hline
\end{tabular}

R. Bras. Zootec., v.32, n.1, p.106-114, 2003 
pressão de aspiração contínua, os folículos menores, de $3 \mathrm{~mm}$, não contados, podem ter sido aspirados, ocorrendo em alguns animais taxa de recuperação superior a $100 \%$. Em um animal do tratamento 2, (FSH+eCG) que apresentou uma resposta acima da média (52 ovócitos coletados), foram contados apenas 22 folículos; isto pode ter ocorrido em conseqüência de os folículos maiores ocultarem os menores, dificultando a contagem correta.

Porcentagem de ovócitos morfologicamente viáveis: não foram encontradas diferenças significativas entre o número de ovócitos viáveis, segundo sua qualidade morfológica, para os três tratamentos, considerando-se as idades de coleta $(p>0,05)$.

$O$ fato de não haver diferença significativa entre a quantidade de ovócitos morfologicamente viáveis (Tabela 3) pode indicar que a sincronização da onda folicular permitiu que todos os ovócitos estivessem na mesma fase fisiológica, não havendo diferença entre os estimulados ou não.

O percentual de ovócitos viáveis, neste trabalho, baseado em sua morfologia, foi de 51 a $66 \%$. Estes resultados estão de acordo com os reportados por Palma et al. (1993), Rick et al. (1996), Presicce et al. (1997) e Majerus et al. (1999).

Na Figura 1, apresenta-se a distribuição da qualidade dos ovócitos dentro de cada tratamento (FSH, $\mathrm{FSH}+\mathrm{eCG}$ e controle), em cada idade (10, 11 e 12 meses de idade). Apesar de em muitas classificações não se considerar viável o COC com cumulus expandido (Presicce et al., 1997; Majerus et al., 1999; Taneja et al., 2000), este trabalho o considera viável

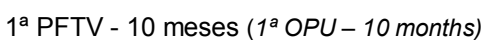

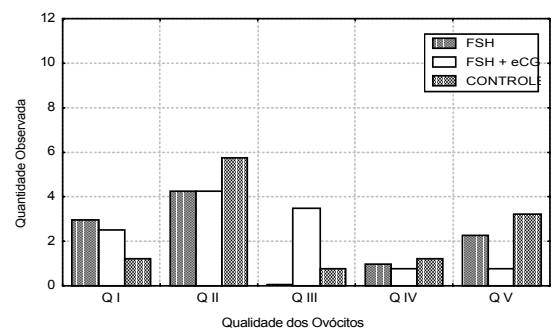

$2^{\mathrm{a}}$ PFTV - 11 meses (2a OPU - 11 months)

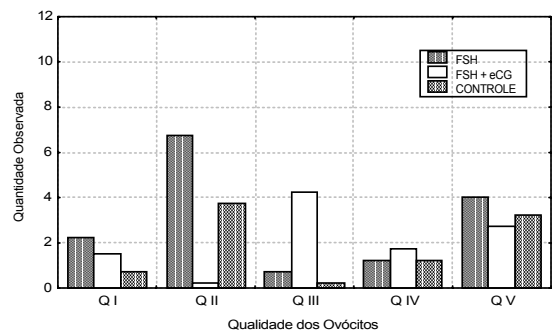

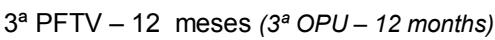

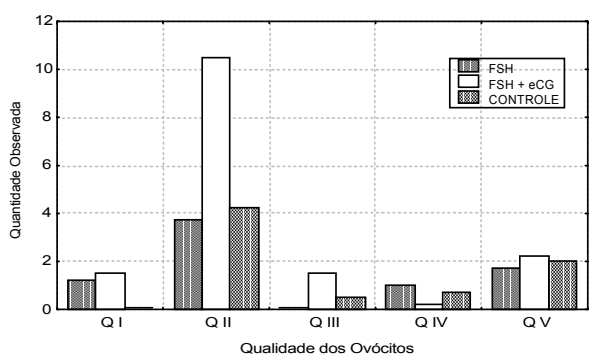

Figura 1 - Quantidade de ovócitos observados de acordo com a qualidade (Q I, Q II, Q III, Q IV e Q V) para cada tratamento ( $\mathrm{FSH}, \mathrm{FSH}+\mathrm{eCG}$ e Controle), em cada idade (10, 11 e 12 meses).

Figure 1 - Quantity of oocytes recovery according whith the quality (Q I, Q II, Q III, QIV e Q V) to each treatment (FSH, FSH + eCG and Control), in each age (10, 11 and 12 months).

Tabela 3 - Valores médios (\%) e desvios-padrão (DP) da quantidade de ovócitos viáveis nas três idades (10, 11 e 12 meses) para cada tratamento (FSH; FSH + eCG e controle)

Table 3 - Means (\%) and standard deviations (SD) of the quantity of viables oocytes in the 3 ages (10, 11 and 12 months) to each treatment (FSH; FSH + eCG and control)

\begin{tabular}{lccccc}
\hline $\begin{array}{l}\text { Tratamento } \\
\text { Treatment }\end{array}$ & $\begin{array}{c}10 \text { meses média \% (DP) } \\
10 \text { months mean \% (SD) }\end{array}$ & $\begin{array}{c}11 \text { meses média \% (DP) } \\
11 \text { months mean \% (SD) }\end{array}$ & $\begin{array}{c}12 \text { meses média \% (DP) } \\
12 \text { months mean \% (SD) }\end{array}$ & $\begin{array}{c}\text { Média total \% (DP) } \\
\text { Total mean \% (SD) }\end{array}$ \\
\hline $\begin{array}{l}\text { FSH }^{1} \\
F S H\end{array}$ & $72,39(19,046)$ & $58,54(20,975)$ & $47,80(35,123)$ & 59,58 & $(25,806)$ \\
$\begin{array}{l}F S H+\mathrm{eCG}^{2} \\
F S H+e C G\end{array}$ & $88,94(12,983)$ & $50,00(40,824)$ & $59,13(44,726)$ & 66,02 & $(36,711)$ \\
$\begin{array}{l}\text { Controle } \\
\text { Control }\end{array}$ & $63,46(16,856)$ & $46,04(42,248)$ & $45,04(33,165)$ & 51,51 & $(30,696)$ \\
\hline
\end{tabular}

Análise de variância com medidas repetidas no tempo (repeated measure analysis of variance), $p>0,05$.

1120 UI de FSH (Folltropin ${ }^{\circledR}$ ), divididos em quatro aplicações im a cada 12 horas, iniciado no D6 (120 IU of FSH (Folltropin ${ }^{\circledR}$ ), divided into four im applications every 12 hours, starting on D6).

2120 UI de FSH (Folltropin $\AA$ ), em uma única aplicação subcutânea (sc), mais 300 UI de eCG (Foligon $\left.{ }^{\circledR}\right)$ sc, no D6 (120 IU of FSH (Folltropin $\left.{ }^{\circledR}\right)$, subcutaneously (sc) in one single administration, plus $300 \mathrm{IU}$ of sc eCG (Foligon $\left.{ }^{\circledR}\right)$, on D6).

${ }^{3}$ sem tratamento estimulatório (control - no stimulating treatment). 
por ter-se sincronizado, previamente, a onda folicular, não sendo este ovócito proveniente de ondas foliculares anteriores.

A qualidade morfológica dos ovócitos está altamente relacionada com sua competência em evoluir para um embrião (Hazeleger \& Stubbings, 1992; Hawk \& Wall, 1994; Kelly et al., 1997; Khurana \& Niemann, 2000; Wit et al., 2000; Wit \& Kruip, 2001).

No experimento, $9,54 \%$ dos ovócitos recuperados eram desnudos ou com poucas células do cumulus (QIV); por isso, pode-se considerar que a press69ão de aspiração (10-15 mL de água/minuto) e o diâmetro interno da agulha $(17 \mathrm{G})$ estavam apropriados. Malard (2000), puncionando através de laparotomia bezerras Nelore e utilizando a mesma classificação dos ovócitos, obteve 35,6 e $32,16 \%$ (controle e estimulado, respectivamente) de ovócitos desnudos. Segundo Hashimoto et al. (1999) e Bols et al. (1996), a combinação do diâmetro da agulha e da pressão de aspiração é crucial para a recuperação de COC de boa qualidade.

Neste estudo não foi encontrada diferença com relação ao número de ovócitos viáveis entre os animais estimulados e não estimulados, ao contrário do relatado por Presicce et al. (1995 e 1997) e Kuwer et al. (1999), em que o tratamento hormonal estimulatório com gonadotrofinas aumentou a qualidade morfológica do ovócito e, conseqüentemente, o desenvolvimento de embriões em bezerras pré-púberes.

Correlação entre número de folículos $\geq 3 \mathrm{~mm}$ a serem aspirados e número de ovócitos recuperados e viáveis colhidos ao longo do experimento: quando foi analisado o número de ovócitos recuperados aos 10, 11 e 12 meses, em relação ao número de folículos $\geq 3 \mathrm{~mm}$ a serem aspirados, achou-se uma correlação positiva $(r=0,66 ; r=0,72$ e $r=0,80$, respectivamente; $p<0,05)$, indicando que, quanto maior o número de folículos, maior o número de ovócitos recuperados. Da mesma forma, ao analisar-se o número de ovócitos viáveis aos 10,11 e 12 meses, em relação ao número de folículos $\geq 3 \mathrm{~mm}$ a serem aspirados, achou-se uma correlação positiva $(\mathrm{r}=$ 0,$76 ; r=0,56$ e $r=0,60$, respectivamente; $p<0,05$ ), indicando que quanto maior o número de folículos, maior o número de ovócitos viáveis. Hashimoto et al. (1999) também demonstraram que o número de ovócitos recolhidos transvaginalmente aumenta quando aumenta o número de folículos a serem aspirados $(\mathrm{P}<0,001)$.

Tamanho dos ovócitos: dos 402 ovócitos coletados, 53 foram desnudados e medidos, incluindo $1^{\text {a }}$ PFTV - 10 meses (1a OPU - 10 months)

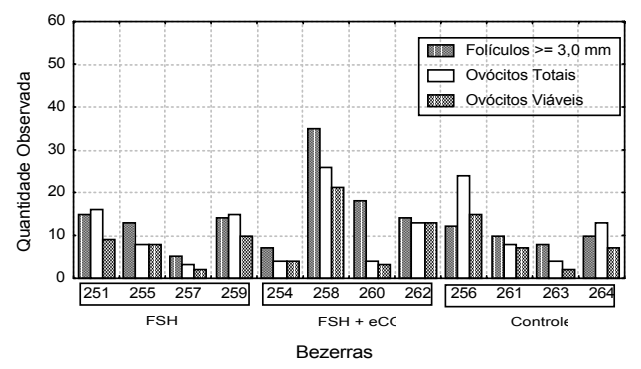

$2^{\mathrm{a}}$ PFTV - 11 meses (2a OPU - 11 months)

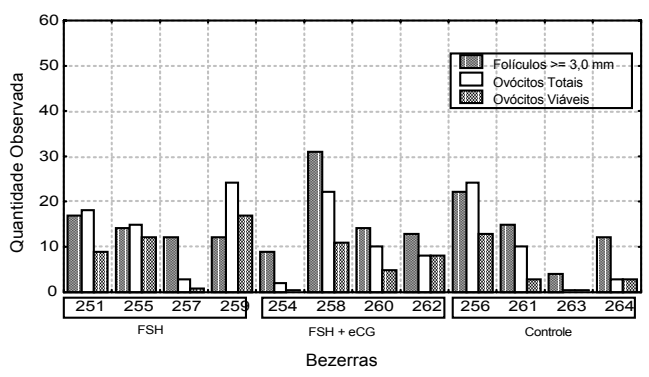

$3^{\mathrm{a}}$ PFTV - 12 meses (3a OPU - 12 months)

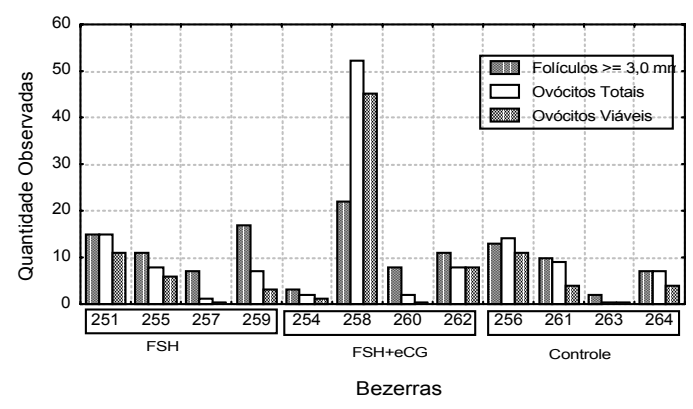

Figura 2 - Quantidade de folículos $\geq 3,0 \mathrm{~mm}$, de ovócitos recuperados e de ovócitos viáveis por animal, de acordo com o tratamento (FSH, FSH + eCG e Controle).

Figure 2 - Quantity of follicles $\geq 3 \mathrm{~mm}$, of oocytes recovery and of viables oocytes according with the treatment (FSH, FSH + eCG and Control).

a zona pelúcida, encontrando-se um diâmetro médio de 153,38 $( \pm 8,06) \mu \mathrm{m}$. Já Gandolfi et al. (1998), estudando bezerras holandesas, obteve uma média de $118( \pm 1,1) \mu \mathrm{m}$ para bezerras de $10-14$ semanas de idade e $122,8( \pm 0,7) \mu \mathrm{m}$ para ovócitos de ovários de vacas de abatedouro. A média do diâmetro do ovócito é menor nas bezerras $(<120 \mu \mathrm{m})$ do que nas vacas $(>120 \mu \mathrm{m})$, mesmo quando as bezerras são hormonalmente estimuladas (Kuwer et al., 1999).A grande diferença no tamanho dos ovócitos, encontrada por outros autores, está relacionada com a técnica 
empregada. Neste trabalho, a zona pelúcida está incluída, nos demais trabalhos, mede-se o diâmetro interno do ovócito, excluindo-se a zona pelúcida (Fair et al., 1995). Dode et al. (2000), ao medirem ovócitos de zebuínos adultos (ovários de abatedouro), utilizando a mesma técnica do presente trabalho, encontraram um diâmetro médio de $159,8( \pm 9,2)$ a $164,4( \pm 9,1)$ para folículos $\geq 3 \mathrm{~mm}$.

Variação individual das doadoras: uma variação individual entre as doadoras foi observada com relação ao número de folículos $\geq$ a $3 \mathrm{~mm}$, ovócitos recuperados e ovócitos viáveis, dentro do mesmo tratamento, influenciando o resultado das análises (Figura 2).

A grande variabilidade dos resultados foi similar ao que ocorre em animais adultos em resposta a regimes de superovulação comparáveis e também já descritos para animais pré-púberes por Armstrong et al. (1992); Majerus et al. (1999); Malard (2000) e Taneja et al. (2000). Como em todos os tratamentos, as bezerras tiveram suas ondas foliculares sincronizadas e desde o nascimento receberam o mesmo manejo e alimentação. Esta variabilidade deve ser devido a diferenças genéticas ou a um efeito maternal exercido durante o período de oogenesis no útero (Armstrong et al., 1992).

\section{Conclusões}

É possível colher ovócitos pela PFTV de bezerras pré-púberes da raça Nelore (Bos taurus indicus) a partir de 10 meses de idade com peso médio de $167,5 \mathrm{~kg}$ (142 a $205 \mathrm{~kg}$ ) e escore corporal médio de 2,5 (escala de 1 a 5), desde que se façam as devidas adequações no sistema de punção utilizado.

$\mathrm{O}$ uso de FSH e FSH + eCG não aumentou significantemente a quantidade de folículos $\geq 3 \mathrm{~mm}$ no momento da PFTV, assim como dos ovócitos recuperados e viáveis aos 10, 11 e 12 meses de idade.

O número de folículos maiores ou iguais a $3 \mathrm{~mm}$, no momento da colheita, está relacionado positivamente com o número de ovócitos recuperados e viáveis.

\section{Literatura Citada}

ADAMS, G.P.; BROGLIATTI, G.M.; SALAMONE, D.F. et al. Superstimulatory response and oocyte collection in calves. Theriogenology, v.45, p.281, 1996.

ARMSTRONG, D.T.; HOLM, P.; IRVINE,B. et al. Pregnancies and live birth from in vitro fertilization of calf oocytes collected by laparoscopic follicular aspiration.

R. Bras. Zootec., v.32, n.1, p.106-114, 2003
Theriogenology, v.38, n.4, p.667-678, 1992.

ARMSTRONG, D.T.; IRVINE, B.J.; EARL, C.R. et al. Gonadotropin stimulation regimens for follicular aspiration and in vitro embryo production from calf oocytes. Theriogenolog $y$, v.42, p.1227-1236, 1994.

ARMSTRONG, D.T.; KOTARAS, P.J.; EARL, C.R. Advances in production of embryos in vitro from juvenile and prepubertal oocytes from the calf and lamb. Reproduction Fertility and Development, v.9, n.3, p.333-339, 1997.

BARROS, C.M. ; FIGUEIREDO, R.A.; PINHEIRO, O.L. Estro, ovulação e dinâmica folicular em zebuínos. Revista Brasileira de Reprodução Animal, v.19, n.1-2, p.9-22, 1995.

BOLS, P.E.J.; VAN SOOM, A.; YSEBAERT, M.T. et al. Effects of aspiration vacuum and needle diameter on cumulus oocyte complex morphology and developmental capacity of bovine oocytes. Theriogenolgy, v.45, n.5, p.1001-1014, 1996.

BOLS, P.E.J.; VAN DE VELDE, A.; RIESEN, J. et al. Pregnancies from prepubertal heifers following repeated oocyte collection and IVF between 6 to 12 months of age. Theriogenology, v.51, n.1, p.298, 1999.

BROGLIATTI, G. M.; ADAMS, G. P. Ultrasound-guided transvaginal oocyte collection in prepubertal calves. Theriogenology, v.45, n.6, p.1163-76, 1996.

BROGLIATTI, G.M.; FURNUS, C.C.; DE MATOS, D.G. et al. In vitro fertilization program in prepuberal Brangus calves and later embryo collection. Theriogenology, v.51, n.1, p.313, 1999.

CROWDER, M.J.; HAND, D.J. Analysis of repeated measures. monographs on statistics and applied probability. London: Chapman \& Hall, 1996. v. 41, 257p.

DAMIANI, P.; FISSORE, R.A.; CIBELLI, J.B. et al. Evaluation of developmental competence, nuclear and ooplasmic maturation of calf oocytes. Molecular Reproduction and Development, v.45, n.4, p.521-534, 1996.

DESJARDINS, C.; HAFS, H.D. Maturation of bovine female genitalia from birth through puberty. Journal of Animal Science, v.28, p.502-507, 1969.

DODE, M.A.N.; RODOVALHO, N.C.; UENO, V.G et al. Efeito do tamanho do folículo na maturação nuclear e citoplasmática de ovócitos de fêmeas zebuínas. Pesquisa Agropecuária Brasileira., v.35, n.1, p.207-214, 2000.

EARL, C.R.; FRY, R.C.; MACLELLAN, et al. In vitro fertilization and developmental potential of prepubertal calf oocytes. In: GAMETES DEVELOPMENT AND FUNCTION, 1998, Rome. Anais... Rome: Serono Symposia, 1998. p.115137.

ERICKSON, B.H. Development and radioresponse of the prenatal bovine ovary. Journal of Reproduction and Fertility, v.11, p.97-105, 1966a.

ERICKSON, B.H. Development and senescence of the postnatal bovine ovary. Journal of Animal Science, v.25, p.800$805,1966 \mathrm{~b}$.

FAIR, T.; HYTTEL, P.; GREVE, T. Bovine oocyte diameter in relation to maturational competence and transcriptional activity. Molecular Reproduction and Development, v.42, p.437-442, 1995.

FRY, R.C.; SIMPSON, T.L.; SQUIRES, T.J. Ultrasonically guided transvaginal oocyte recovery from calves treated with or without GnRH. Theriogenology, v.49, n.6, p.10771082, 1998.

GIRDEN, E.R. ANOVA: repeated measures. Series: Quantitative Applications in the Social Sciences, SAGE University Paper, 1992. 76p.

GOSDEN, R.G. Biology and technology of primordial follicle 
development. In: GAMETES DEVELOPMENT AND FUNCTION, 1998, Rome. Anais... Rome: Serono Symposia, 1998. p.71-83.

HASHIMOTO, S.; TAKAKURA, R.; KISHI, M. et al. Ultrasound-guided follicle aspiration: the collection of bovine cumulus-oocyte complexes from ovaries of slaughtered or live cows. Theriogenology, v.51, n.4, p.757-765, 1999.

HAWK, H.W.; WALL, R.J. Improved yields of bovine blastocysts from in vitro-produced oocytes. I. Selection of oocytes and zygotes. Theriogenology, v.41, p.1571-1583, 1994.

HAZELEGER, N.L.; STUBBINGS, R.B. Developmental potential of selected bovine oocyte cumulus complexes. Theriogenology, v.37, n.1, p.219, 1992.

IRVINE, B.; ARMSTRONG, D.T.; EARL, C. et al. Follicle development and oocyte recovery from calves with repeated gonadotropin stimulation and follicular aspiration. Theriogenology, v.39, n.1, p.237, 1993.

KAJIHARA, Y.; BLAKEWOOD, E. G.; MYERS, M.W. et al. In vitro maturation and fertilization of follicular oocytes obtained from calves. Theriogenology, v.35, n.1, p.220, 1991.

KELLY, J.; EARL, C.; ROWE, J. et al. Blastocyst production rates from different grades of oocytes collected from 2 to 3 month old calves. Theriogenology, v.47, n.1, p.291,1997.

KHATIR, H.; LONERGAN, P.; CAROLAN, C. et al. Prepubertal bovine oocyte: a negative model for studying oocyte developmental competence. Molecular Reproduction and Development, v.45, n.2, p.231-239; 1996.

KHURANA, N.K.; NIEMANN, H. Effects of oocyte quality, oxigen tension, embryo density, cumulus cells and energy substrates on cleavage and morula/blastocyst formation of bovine embryos. Theriogenology, v.54, n.5, p.741-56, 2000.

KUWER, A.; LEMME, E.; NIEMANN, H. Developmental capacity of cumulus oocyte complexes collected from prepubertal cattle with and without gonadotropin stimulation employing ultrasound-guided follicular aspiration. Theriogenology, v.51, n.1, p.323, 1999.

LÉVESQUE, J.T.; SIRARD, M.A. Proteins in oocytes from calves and adult cows before maturation: relationship with their development capacity. Reproduction, Nutrition and Development, v.34, p.133-139, 1994.

LOHUIS, M.M. Potential benefits of bovine embryo manipulation technologies to genetic improvement programs. Theriogenology, v.43, n.1, p.51-60, 1995.

LOONEY, C.R.; DAMIANI, P.; LINDSEY, B.R. et al. Use of prepuberal heifers as oocyte donors for IVF: effect of age and gonadotropin treatment. Theriogenology, v.43, n.1,p.269, 1995.

MACLELLAN, L.J.; WHYTE, T.R.; MURRAY, A. et al. Superstimulation of ovarian follicular growth with $\mathrm{FSH}$, oocyte recovery, and embryo production from zebu (Bos indicus) calves: effects of treatment with GnRH agonist or antagonist. Theriogenology, v.49, n.7, p.1317-1329, 1998a.

MACLELLAN, L.J.; WHYTE, T.R.; EARL, C.R. et al. Relationship of live weight gain to the response of Zebu heifer calves to estimulation with FSH, oocyte recovery and in vitro embryo development. In: GAMETES DEVELOPMENT AND FUNCTION, 1998, Rome. Anais... Rome: Serono Symposia, 1998b. p.568.

MAJERUS, V.; DE ROOVER, R.; ETIENNE, D. et al. Embryo production by ovum pick up in unstimulated calves before and after puberty. Theriogenology, v.52, n.7, p.1169-1179, 1999.

MALARD, P.F. Coleta, maturação, fecundação e cultivo in vitro de ovócitos de bezerras da raça Nelore de 2 a 3 meses de idade. Belo Horizonte: Universidade Federal de Minas
Gerais, 2000. 47p. Dissertação (Mestrado) - Universidade Federal de Minas Gerais, 2000.

PALMA, G.A.; CLEMENT SENGEWALD, A.; KREFFT, H. In vitro production of cattle embryos from calf oocytes. Theriogenology, v.39, p.278, 1993.

PIETERSE, M.C.; KAPPEN, K.A., KRUIP, T.A.M. et al. Aspiration of bovine oocytes during transvaginal ultrasound scanning ovaries. Theriogenology, v.30, p.751-762, 1988.

PRESICCE, G.A.; JIANG, S.; SIMKIN, M. et al. Oocyte quality and embryo development in prepubertal calves. Biology of Reproduction, v.52 (Suppl 1), p.127, 1995.

PRESICCE, G.A.; JIANG, S.; SIMKIN, M. et al. Age and hormonal dependence of acquisition of oocyte competence for embryogenesis in prepubertal calves. Biology of Reproduction, v.56, n.2, p.386-392, 1997.

RESTLE, J.; POLLI, V.A.; SENNA, D. B. Efeito de grupo genético e heterose sobre a idade e peso a puberdade e sobre o desempenho reprodutivo de novilhas de corte. Pesquisa Agropecuária Brasileira, v.34, n.4, p.701-707, 1999.

REVEL, F; MERMILLOD; P. PEYNOT, N. et al. Low developmental capacity of in vitro matured e fertilized oocytes from calves compared with that of cows. Journal of Reproduction and Fertility, v.103, p.115-120, 1995.

RICK, G.; HADELER, K.G.; LEMME, E. et al. Long-term ultrasound guided ovum pick-up in heifers from 6 to 15 months of age. Theriogenology, v.45, n.1, p.356, 1996.

RODRIGUES, H.D.; KINDER, J.E.; FITZPATRICK, L.A. Treatment with 17 beta-oestradiol does not influence age and weight at puberty in Bos indicus heifers. Animal Reproduction Science, v.56, n.1, p.1-10, 1999.

SEIDEL, G.E.; JR., LARSON, L.L.; SPILMAN, C.H. et al. Culture and transfer of calf ova. Journal of Dairy Science, v.54, p.923-926, 1971.

SILVA, A.E.D.F.; ROMANO, M.A. Alguns aspectos de puberdade maturidade de fêmeas da raça Canchim, Nelore, e meiosangue Canchim e Nelore. In: CONGRESSO BRASILEIRO DE REPRODUÇÃO ANIMAL, 9., 1991, Belo Horizonte. Anais... Belo Horizonte: Colégio Brasileiro de Reprodução Animal, 1991. v.2, p.381.

STUBBINGS, R.B.; WOSIK, C.; SUEPAUL, M. et al. Follicular response of prepubertal calves to repeated stimulation with FSH. Theriogenology, v.37, n.1, p.304, 1992.

TANEJA, M.; BOLS, P.E.J.; VAN DE VELDE, A. et al Developmental competence of juvenile calf oocytes in vitro and in vivo: influence of donor animal variation and repeated gonadotropin stimulation. Biology of Reproduction, v.62, n.1, p.206-213, 2000.

TERVIT, H.R.; McMILLAN, W.H; McGOWAN, L.T. et al. Effect of juvenile calf age on follicular dynamics and in vitro embryo production. Theriogenology, v.47, n.1, p.300, 1997.

WIT, A.A.C.; KRUIP, T.A.M. Bovine cumulus-oocyte-complex-quality is reflected in sensitivity for alpha-amanitin, oocyte-diameter and developmental capacity Animal Reproduction Science, v.65, n.1-2, p.51-65, 2001.

WIT, A.A., WURTH, Y.A.; KRUIP, T.A. Effect of ovarian phase and follicle quality on morphology and developmental capacity of the bovine cumulus-oocyte complex. Journal Animal Science, v.78, n.5, p.1277-1283, 2000. 